\title{
Validation of the adjusted multi-biomarker disease activity score as a prognostic test for radiographic progression in rheumatoid arthritis: a combined analysis of multiple studies
}

\author{
Jeffrey R. Curtis', Michael E. Weinblatt ${ }^{2}$, Nancy A. Shadick ${ }^{2}$, Cecilie H. Brahe ${ }^{3,4}$, Mikkel Østergaard ${ }^{3,4}$,
} Merete Lund Hetland ${ }^{3,4}$, Saedis Saevarsdottir ${ }^{5,6}$, Megan Horton7, Brent Mabey ${ }^{7}$, Darl D. Flake II7,

Rotem Ben-Shachar ${ }^{7}$, Eric H. Sasso ${ }^{8^{*}}$ (I) and T. W. Huizinga ${ }^{9}$

\begin{abstract}
Background: The multi-biomarker disease activity (MBDA) test measures 12 serum protein biomarkers to quantify disease activity in RA patients. A newer version of the MBDA score, adjusted for age, sex, and adiposity, has been validated in two cohorts (OPERA and BRASS) for predicting risk for radiographic progression. We now extend these findings with additional cohorts to further validate the adjusted MBDA score as a predictor of radiographic progression risk and compare its performance with that of other risk factors.

Methods: Four cohorts were analyzed: the BRASS and Leiden registries and the OPERA and SWEFOT studies (total $N=953$ ). Treatments included conventional DMARDs and anti-TNFs. Associations of radiographic progression ( $\Delta$ TSS) per year with the adjusted MBDA score, seropositivity, and clinical measures were evaluated using linear and logistic regression. The adjusted MBDA score was (1) validated in Leiden and SWEFOT, (2) compared with other measures in all four cohorts, and (3) used to generate curves for predicting risk of radiographic progression.

Results: Univariable and bivariable analyses validated the adjusted MBDA score and found it to be the strongest, independent predicator of radiographic progression ( $\triangle T S S>5$ ) compared with seropositivity (rheumatoid factor and/or anti-CCP), baseline TSS, DAS28-CRP, CRP SJC, or CDAI. Neither DAS28-CRP, CDAI, SJC, nor CRP added significant information to the adjusted MBDA score as a predictor, and the frequency of radiographic progression agreed with the adjusted MBDA score when it was discordant with these measures. The rate of progression $(\Delta T S S>5)$ increased from $<2 \%$ in the low (1-29) adjusted MBDA category to $16 \%$ in the high (45-100) category. A modeled risk curve indicated that risk increased continuously, exceeding $40 \%$ for the highest adjusted MBDA scores.

(Continued on next page)
\end{abstract}

\footnotetext{
* Correspondence: esasso@myriad.com

${ }^{8}$ Crescendo Bioscience, Inc., 180 Kimball Way, South San Francisco, CA, USA

Full list of author information is available at the end of the article
}

\section{$\triangle B M C$}

(c) The Author(s). 2021 Open Access This article is licensed under a Creative Commons Attribution 4.0 International License, which permits use, sharing, adaptation, distribution and reproduction in any medium or format, as long as you give appropriate credit to the original author(s) and the source, provide a link to the Creative Commons licence, and indicate if changes were made. The images or other third party material in this article are included in the article's Creative Commons licence, unless indicated otherwise in a credit line to the material. If material is not included in the article's Creative Commons licence and your intended use is not permitted by statutory regulation or exceeds the permitted use, you will need to obtain permission directly from the copyright holder. To view a copy of this licence, visit http://creativecommons.org/licenses/by/4.0/ The Creative Commons Public Domain Dedication waiver (http://creativecommons.org/publicdomain/zero/1.0/) applies to the data made available in this article, unless otherwise stated in a credit line to the data. 


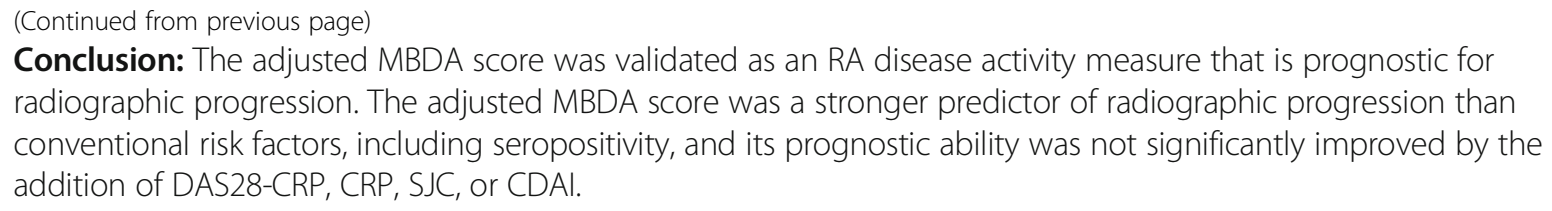

Keywords: Biomarker, Rheumatoid arthritis, Disease activity, Radiographic progression, Risk prediction

\section{Introduction}

The goals of managing patients with rheumatoid arthritis (RA) are to minimize inflammation, joint damage, and disability. To achieve these goals, RA disease activity should be quantitatively assessed on a regular basis, with treatment adjusted as needed to achieve remission or the lowest possible level of disease activity $[1,2]$. Ideally, this strategy should be initiated from disease onset because the disability associated with joint damage is irreversible.

Preventing new joint damage remains an important goal for RA patient management. Although biologic agents have reduced the amount of joint damage progression observed in clinical trials and in practice, progression occurs in some patients receiving biologic therapy [3-8]. In addition, biologic therapy is not always initiated promptly in patients with inadequate response to a conventional disease-modifying anti-rheumatic drug (DMARD) [9]. Remission can be difficult to achieve, even with treat-to-target strategies [10], in part from difficulty distinguishing between inflammatory and noninflammatory symptoms [11]. Moreover, joint damage can occur with synovitis that is inapparent on physical examination [12]. In clinical trials, joint damage is measured with radiographs and specialized scoring systems, such as the van der Heijde modified Sharp score [13]. An increase of five Sharp units per year was found to be the minimal clinically important difference needed for rheumatologists to change therapy [14], and increase $>5$ units per year has been used to define rapid radiographic progression in clinical trials [15]. In clinical practice, joint damage progression is not usually assessed quantitatively. It could be useful to have a convenient way to assess the risk for new joint damage without X-rays or advanced imaging.

The probability of developing new joint damage is related to several factors, including a history of joint damage, serologic status, and the level of RA disease activity. Of these factors, only disease activity can be reliably influenced by treatment. Most rheumatologists in the US do not routinely use a composite disease activity measure with joint counts to assess RA disease activity, as the required assessments are time-consuming [16]. Moreover, the components of these measures have shortcomings that limit their ability to predict radiographic progression. Joint counts and global assessments are partially or entirely subjective and can vary between assessors [17], and Creactive protein (CRP) and erythrocyte sedimentation rate (ESR) are objective but are non-specific and insensitive $[18,19]$. In a study of 9135 patients with clinically active RA in rheumatology practices in the US, neither CRP nor ESR was elevated in the majority of patients, including $46 \%$ of those with a high Clinical Disease Activity Index (CDAI) [20]. These considerations demonstrate the need for an accurate, objective tool that measures RA disease activity and is prognostic for new joint damage.

The multi-biomarker disease activity (MBDA) blood test measures 12 serum biomarkers, including CRP, to generate a score on a scale of 1-100 that represents the level of disease activity in adult patients with RA [21, $22]$. The MBDA score has categories of low $(<30)$, moderate (30-44), and high $(>44)$ disease activity [21, 22]. Change in MBDA score correlates with change in clinical disease activity [23]. When assessing change from a moderate or high MBDA score to a later score, the minimally important difference is 8 points [24]. In 2019, the MBDA score was the subject of a systematic review and meta-analysis [25], and the American College of Rheumatology (ACR) disease activity measures working group concluded that the MBDA score was one of 11 RA disease activity measures that fulfilled the minimum standard for regular use [26].

In single-cohort studies, a low MBDA score was associated with little or no radiographic progression over the following the year and high MBDA scores conferred the highest risk for progression [6, 27-29]. The MBDA score was a stronger predictor of risk for radiographic progression than conventional disease activity measures in these studies and in a combined analysis of three cohorts [30].

A recent study of over 300,000 RA patients developed and validated a method for modifying the original MBDA score to adjust it for the effects of the age, sex, and serum leptin concentration, used as a surrogate for adiposity [31]. In an analysis of cohorts from OPERA, a study of treatment-naïve patients with recent onset RA, and BRASS, a US registry of patients with predominantly longstanding RA, the adjusted MBDA score was a stronger predictor of the risk for radiographic progression than the original MBDA score and both were stronger predictors than seropositivity and several conventional 
measures of disease activity [31]. That analysis is the only one to date which examined the risk for radiographic progression using the adjusted MBDA score.

The goal of the present study was to validate the adjusted MBDA score as a prognostic test for radiographic progression in two cohorts that have not been evaluated previously with the adjusted MBDA score. These two cohorts were then pooled with the previously analyzed OPERA and BRASS cohorts to create a large, diverse combined cohort from two prospective studies and two registries to further evaluate the performance of the adjusted MBDA score, to compare it with other measures, and to generate a risk curve for predicting the probability of radiographic progression in individual patients based on the adjusted MBDA score.

\section{Methods}

\section{Patient cohorts}

Four cohorts were selected for this analysis because of their size (>100 patients) and availability of requisite clinical, radiographic, and MBDA score patient-level data. These cohorts (Leiden, OPERA, SWEFOT, and BRASS) include patients receiving non-biologic and biologic DMARDs. They have not all been pooled previously to form a single cohort, and the Leiden and SWEFOT cohorts have not been analyzed previously with the adjusted MBDA score. Three of these cohorts (Leiden, OPERA, SWEFOT) have been previously analyzed using the original, unadjusted MBDA score as a prognostic for radiographic progression, both individually $[6,28,29]$ and in a combined analysis for which they were selected by a literature review conducted in October 2018 [30]. Cohorts from BRASS and OPERA were previously used to validate the adjusted MBDA score as a prognostic for radiographic progression [31]. For consistency, the same cohorts were included here.

\section{Clinical and laboratory measures}

The present analyses used patient-level data that were collected by the respective parent study (OPERA or SWEFOT) or registry (Leiden or BRASS). Swollen and tender joint counts (SJC, TJC) were based on 28 joints. The Disease Activity Score with 28 joints using CRP (DAS28-CRP) categories employed here for remission/ low ( $\leq 2.67$, termed "low" hereafter), moderate ( $>2.67$ to 4.09), and high (>4.09) disease activity have been established specifically for DAS28-CRP with thresholds that are lower than those for DAS28-ESR [32]. Because DAS28 results in the OPERA parent trial used ESR, CRP data from OPERA were used to generate DAS28-CRP values for use here. Physician global assessment data were unavailable for the Leiden cohort, so CDAI was analyzed only for the OPERA, SWEFOT, and BRASS cohorts. CDAI categories were low $(\leq 10)$, moderate $(>10$ to 22), and high (>22). CRP data were from the respective clinical laboratories used by OPERA, SWEFOT, and BRASS. CRP data for Leiden came from the highsensitivity measurement in the MBDA test. The CRP categories employed here were low $(\leq 3.0 \mathrm{mg} / \mathrm{L})$, moderate $(>3.0$ to $10 \mathrm{mg} / \mathrm{L})$, and high $(>10 \mathrm{mg} / \mathrm{L})$ and are based on the threshold used in the ACR/EULAR definition of Boolean remission (CRP $\leq 10 \mathrm{mg} / \mathrm{L}$ ) [33] and are consistent with categories used in previous reports [15, 28-30, 34-37]. SJC categories were low $(\mathrm{SJC}=0)$, moderate $(\mathrm{SJC}=1$ to 9$)$, and high $(\mathrm{SJC} \geq 10)$ and were chosen to emphasize the lower end of the SJC spectrum, where elevated adjusted MBDA scores are of special interest. Serologic status was defined as positive if a patient had tested positive for rheumatoid factor (RF), anti-cyclic citrullinated peptide (CCP) antibodies or both in the clinical laboratory of the respective cohort, and negative if the patient had tested negative for both RF and anti-CCP antibodies.

\section{Radiographic measures}

Radiographs of the hands, wrists, and feet were obtained at baseline and 1 year for Leiden, OPERA, and SWEFOT. Radiographs of the hands and wrists were obtained at baseline and approximately 2 years later for BRASS. For each cohort, radiographs were assessed with the van der Heijde-modified total Sharp score (TSS) by unique, independent assessors who were blinded to clinical data and MBDA scores. As validated previously, TSS data from BRASS were multiplied by $1.6(448 / 280)$ to normalize for the number of joints that would have been scored if radiographs had also included the feet, and they were normalized to a per 365 days rate of change $(\Delta)$ in TSS for each patient, based on the time between paired radiographs of a patient [31]. Radiographic progression was defined as $\triangle \mathrm{TSS}>5$ units per year unless specified otherwise.

\section{Multi-biomarker disease activity score measurement}

For each cohort, MBDA scores were determined previously using serum samples that had been collected by the parent study or registry and shipped frozen to the laboratory of the Crescendo Bioscience in South San Francisco, CA, for testing, as has been reported [6, 28, 29, 31]. Serum concentrations of 12 biomarkers (vascular cell adhesion molecule 1, epidermal growth factor, vascular endothelial growth factor, interleukin 6, TNF receptor type I, matrix metalloproteinase (MMP) 1, MMP-3, bone glycoprotein 39 (YKL-40), leptin, resistin, serum amyloid A, and CRP) were measured by electroluminescence-based multiplexed sandwich immunoassays (Meso Scale Discovery, Rockville, MD, USA) and used to determine the original MBDA score with a validated algorithm [22]. All testing used the same types of reagents and instrument as are used for the 
Vectra $^{\bullet}$ test, which has been commercially available in the USA since 2010. All MBDA scores were determined after the corresponding clinical and radiographic assessments were complete and without knowledge of those results.

For the present study, original MBDA scores were adjusted for age, sex, and leptin concentration using a validated algorithm [31]. The original MBDA score and the adjusted MBDA score both have a scale of $1-100$, disease activity categories of low $(<30)$, moderate (30-44) and high (>44), and a minimally important difference of 8 points [31]. This adjustment has been in the clinical use in the USA since December 2017. Only adjusted MBDA scores were analyzed in this study.

\section{Statistical analyses \\ Univariable analysis of the adjusted MBDA score and other potential risk factors as predictors of radiographic progression}

Univariable analyses of baseline adjusted MBDA score and clinical and demographic variables were performed to predict radiographic progression over 1 year in the Leiden and SWEFOT combined cohorts and in the four cohorts combined. Linear regression models were developed to predict continuous radiographic progression ( $\Delta \mathrm{TSS})$, where coefficient estimates, 95\% confidence intervals (CI), and $p$ values were reported. Radiographic progression was analyzed as a binary outcome $(\Delta \mathrm{TSS}>5)$ using logistic regression models, where odds ratios, $95 \% \mathrm{CI}$, and $p$ values were reported. In univariable and bivariable (see below) analyses, CRP in $\mathrm{mg} / \mathrm{L}$ was treated as the natural logarithm $(\ln )$ of $(C R P+1)$. All models contained a random effect on cohort, and $p$ values were derived from likelihood ratio tests. As risk discrimination is considered a more appropriate metric for assessing performance of a prognostic test, sensitivity, specificity, positive predictive value, and negative predictive value were not evaluated here [38, 39].

\section{Descriptive analyses of association between radiographic progression and adjusted MBDA score}

A scatter plot was constructed to display the $\triangle$ TSS per year as a function of the adjusted MBDA score at the time of the first radiograph for all patients in the combined cohort. The percentage of patients with $\triangle T S S>5$ per year was determined for patient groups based on the category of adjusted MBDA score (low, moderate, or high) at the time of the first radiograph for each cohort and the four cohorts combined, without weighting. Rates of progression were also determined for subgroups within the high MBDA category.
Direct comparison of the adjusted MBDA score and conventional measures of disease activity as predictors of radiographic progression

For the four cohorts combined, the ability of the adjusted MBDA score to predict radiographic progression $(\Delta \mathrm{TSS}>5)$ when combined pairwise with each clinical variable was assessed in bivariable analyses using logistic regression models with a random effect on cohort. Odds ratios, 95\% CI, and $p$ values derived from likelihood ratio tests were reported. In a subsequent descriptive analysis, patients were cross-classified into nine subgroups, based on low $(<30)$, moderate $(30-44)$, and high $(>44)$ adjusted MBDA score categories vs. the low, moderate, and high categories of DAS28-CRP, CRP, SJC, and CDAI, respectively, as defined above. The percentage of patients with radiographic progression $(\Delta \mathrm{TSS}>5$ from baseline to 1 year) was determined for each subgroup.

Risk curves to show radiographic progression as a function of adjusted MBDA score as a continuous variable

To estimate the risk of radiographic progression $(\triangle \mathrm{TSS}>$

5 ) as a function of the baseline adjusted MBDA score as a continuous variable, a mixed-effects logistic regression model was fit with radiographic progression as the response variable, the adjusted MBDA score as a predictor with a fixed effect, and the respective cohort of each patient as a predictor with a random effect. The predicted risks for radiographic progression over 1 year and the associated $95 \%$ profile likelihood-based confidence intervals were calculated for all adjusted MBDA scores from 1 to 100. This risk relationship was presented graphically as a curve. Additional risk curves were generated with radiographic progression defined as $\Delta$ TSS $>2,>3$, and $>4$.

\section{Results}

\section{Cohorts and patients}

Four cohorts were analyzed to determine the relationship between the adjusted MBDA score and risk for radiographic progression (Table 1). These cohorts included studies of patients with recent onset, active RA for whom a new treatment was being initiated (OPERA and SWEFOT) and registries of patients, predominantly with established RA, who were receiving ongoing treatment as part of routine care (BRASS and Leiden). Treatments varied between cohorts, according to the respective protocols, and included conventional synthetic DMARDs and biologic DMARDs, which were all TNF inhibitors except for one patient receiving rituximab in the BRASS cohort (Table 1).

\section{Validation of the adjusted MBDA score as a prognostic test for radiographic progression and comparison with other measures}

To validate the adjusted MBDA score independently of its previous validation in the OPERA and BRASS cohorts 


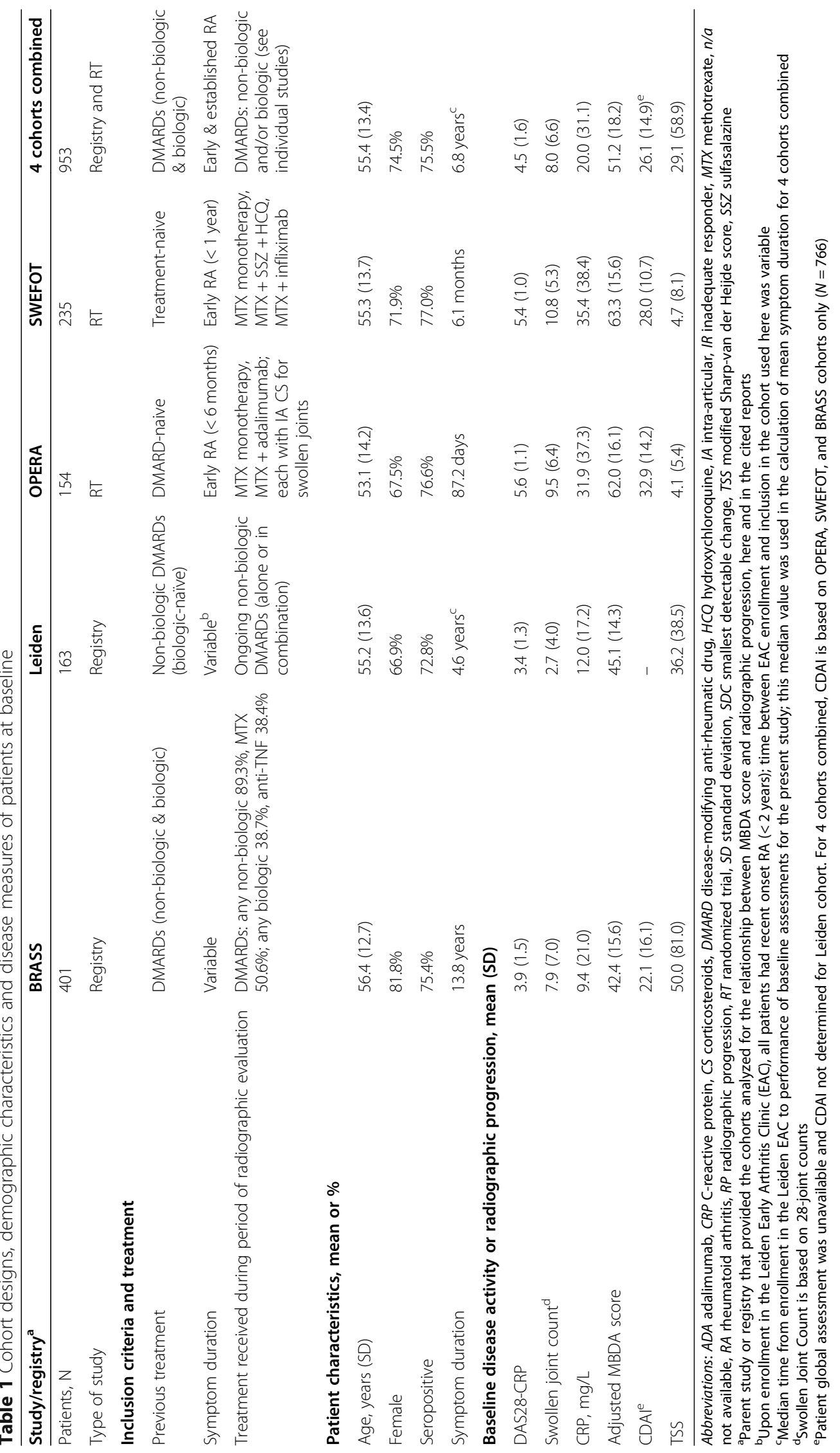


combined [30], univariable analyses were conducted for the Leiden and SWEFOT cohorts combined. The adjusted MBDA score had a strong univariable association with radiographic progression as a binary variable ( $\triangle$ TSS $>5)$, with an OR of $1.05\left(p=5.55 \times 10^{-8}\right)$ (Table 2). Associations were also significant, but less so, for seropositivity $\left(p=1.7 \times 10^{-4}\right)$, CRP (tested as $\ln [\mathrm{CRP}+1], p=$ $0.0018)$, baseline joint damage (tested as TSS) $(p=$ $0.0023)$, and DAS28-CRP $(p=0.035)$. Univariable associations were not significant in Leiden and SWEFOT combined for SJC, CDAI, male sex, or age (Table 2). Univariable analyses treating $\triangle$ TSS as a continuous variable yielded similar results (Table 2, " $\Delta$ TSS continuous"). Similar results were also obtained when analyzing all four cohorts combined $(N=953)$, with smaller $p$ values observed for most measures. Male sex, age, and CDAI were non-significant for both the continuous and the categorical analyses of $\triangle \mathrm{TSS}$ in the four cohorts combined (Table 2).

\section{Relationship between adjusted MBDA score and radiographic progression in individual patients}

A scatter plot of data from the four cohorts combined $(N=953)$ illustrated that most progressors had high MBDA scores, and progression $(\triangle \mathrm{TSS}>5)$ was nearly absent among patients with low-adjusted MBDA scores (Fig. 1). Radiographic progression tended to be not only more frequent but also more severe among patients with higher adjusted MBDA scores.

\section{Relationship between category of adjusted MBDA score and rate of radiographic progression}

While the four cohorts differed in their disease durations and treatment regimens, each one demonstrated a trend where radiographic progression ( $\triangle \mathrm{TSS}>5$ per year) was most frequent in the high adjusted MBDA score category (8.3 to $29.1 \%$ ) and absent or nearly absent among patients with low-adjusted MBDA scores (0 to 4.0\%) (Fig. 2). For the four cohorts combined, radiographic progression was observed in $1.7 \%, 4.4 \%$, and $15.8 \%$ of patients with low $(<30)$, moderate $(30-44)$, or high (> 44 ) adjusted MBDA scores, respectively (Fig. 2). For patients with adjusted MBDA scores of 45-60 $(n=288)$ or $>60(n=296)$, progression was $>3$ Sharp units in 55 (19\%) and in 94 (32\%) patients, respectively; > 5 Sharp units in 28 (9.7\%) and 64 (22\%) patients, respectively; and $>10$ Sharp units in 7 (2.4\%) and 27 (9.1\%) patients, respectively.

Risk ratios $(95 \% \mathrm{CI})$ for $\Delta \mathrm{TSS}>5$ were $2.62(0.59$, 11.6; $p=0.24)$ and $9.37\left(2.34,37.5 ; p=2.65 \times 10^{-6}\right)$ in the moderate and high-adjusted MBDA score categories, respectively, compared to the low category, and 4.47 $\left(2.54,7.87 ; p=5.26 \times 10^{-10}\right)$ for the high category compared to the low and moderate categories combined.
Bivariable analyses to compare the prognostic value of the adjusted MBDA score vs. other measures

Bivariable analyses found that the adjusted MBDA score added statistically significant information to each other measure for predicting risk for radiographic progression $(\Delta \mathrm{TSS}>5)\left(p<1.0 \times 10^{-6}\right.$ for each measure; Supplemental Table 1). By contrast, none of the clinical disease activity measures or CRP added significant predictive information to the adjusted MBDA score $(p>0.05$ for each), indicating that the adjusted MBDA score fully accounted for their predictive information. Significant predictive information was added to the adjusted MBDA score by seropositivity and, to a lesser degree, baseline TSS, but for each, this additional information was less statistically significant than the information the adjusted MBDA score added to them (Supplemental Table 1). No interaction was found between the adjusted MBDA score and any of the other variables, indicating that the effect of the adjusted MBDA score on radiographic progression was consistent across the levels of each variable.

\section{Cross-classification of adjusted MBDA score with conventional disease activity measures to evaluate discordances}

To illustrate the finding of the bivariable analyses-that the adjusted MBDA score predicted progression risk independently of the level of clinical disease activity-patients were grouped into low, moderate, and high categories of DAS28-CRP and, within each category, sub-grouped by category of adjusted MBDA score. The frequency of radiographic progression was very low (0$3.0 \%$ ) when the adjusted MBDA score was low and was highest (13.2-16.8\%) when the adjusted MBDA score was high, regardless of whether the DAS28-CRP was low, moderate, or high (Fig. 3a). This type of trend was not observed across DAS28-CRP categories within adjusted MBDA score categories. Analyses of the four cohorts individually, with cross-classification by DAS28CRP and MBDA score, were generally consistent with the combined cohort analysis, but they should be interpreted with caution, due to the smaller numbers of patients and progressors (Supplemental Figure 1). Similar results were obtained in the combined cohort when cross-classification used categories of CRP, SJC and CDAI (Fig. 3b, c, and d, respectively), except within the low CDAI group, possibly due to the limited number of progressors. Thus, when the adjusted MBDA score was discordant with DAS28-CRP, CRP, SJC, or CDAI, either because the adjusted MBDA score was high and the comparison measure was low or vice versa, the frequency of radiographic progression corresponded more consistently with the category of adjusted MBDA score than the category of DAS28-CRP, CRP, SJC, or CDAI (Fig. 3). 


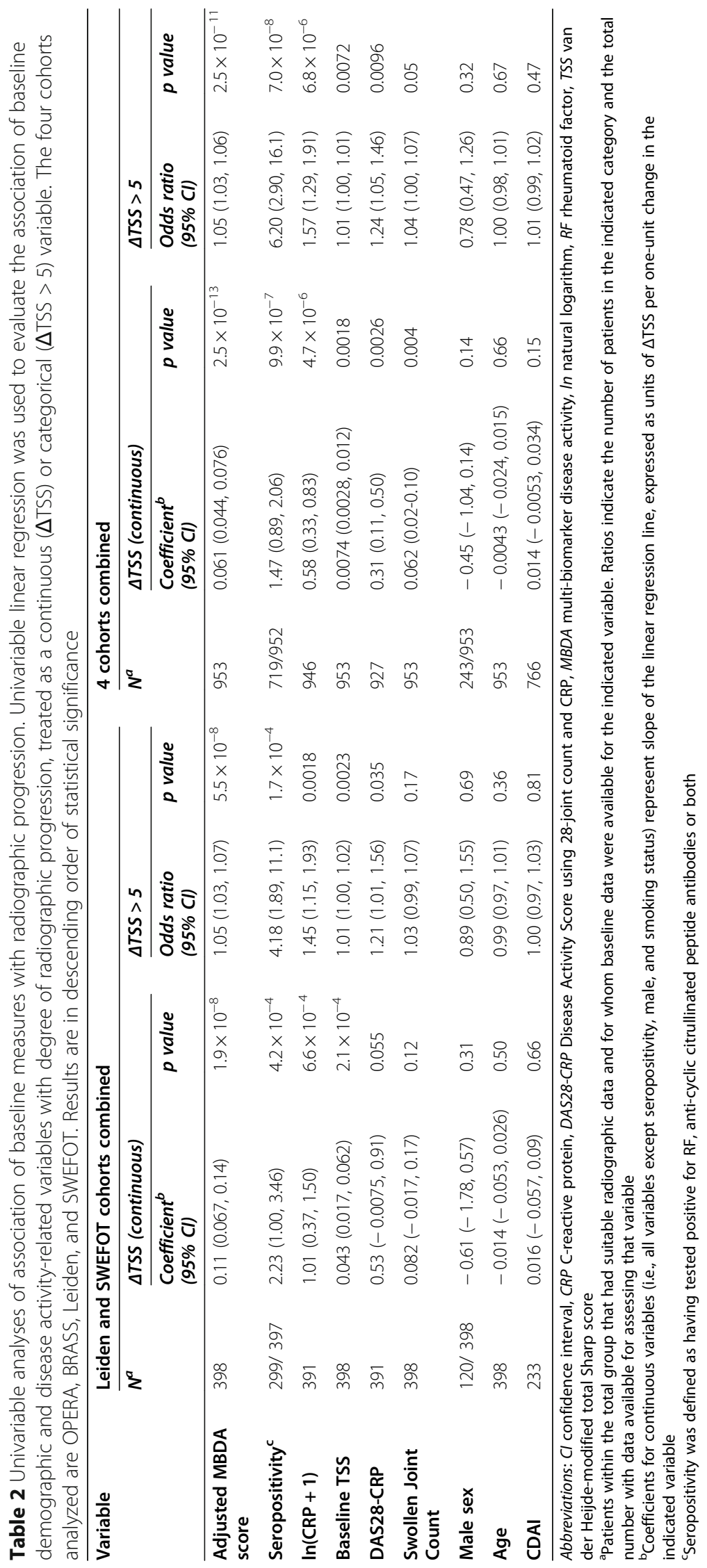




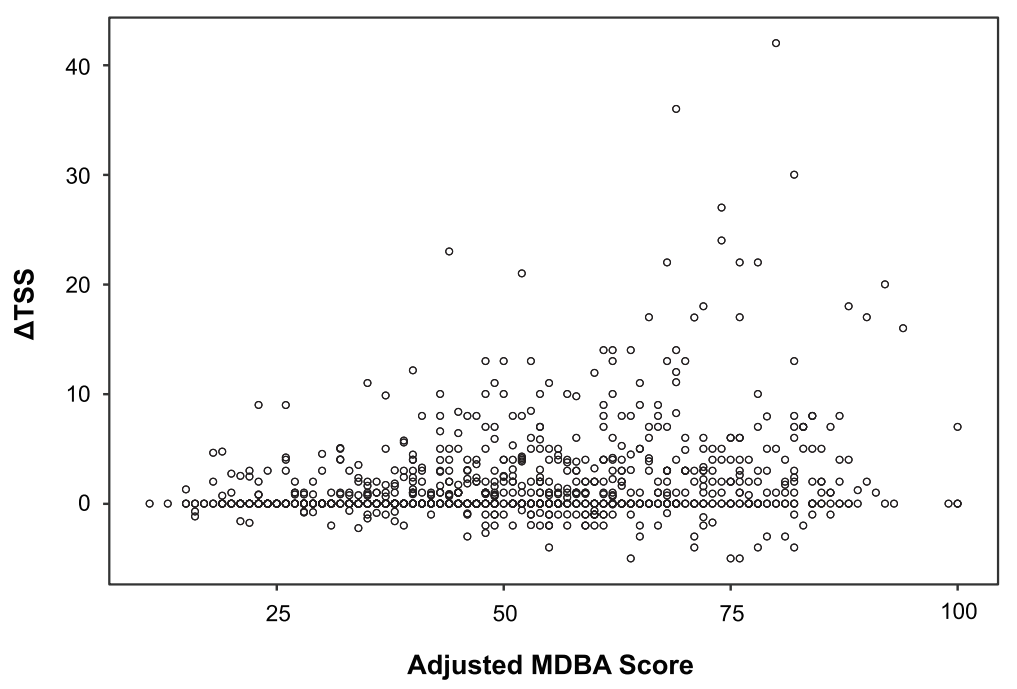

Fig. 1 Relationship between baseline-adjusted MBDA score and radiographic progression over the following year. Each open circle represents a pairing of adjusted MBDA score and change in van der Heijde-modified Sharp score ( $\Delta$ TSS) in the combined cohort $(N=953)$. $\Delta$ TSS $=0$ for 474 patients. MBDA multi-biomarker disease activity

Risk curves for the MBDA score as a continuous variable for predicting risk of radiographic progression

Because the adjusted MBDA score was the strongest single, independent predictor of radiographic progression (Table 2, Supplementary Table 1), we used logistic regression in the combined cohort to generate a risk curve for radiographic progression $(\Delta \mathrm{TSS}>5)$. The probability of progression was lowest when the adjusted MBDA score was low, and it increased continuously as the adjusted MBDA score increased, with an upswing as the score entered the high range (>44) (Fig. 4a). Among the highest adjusted MBDA scores, the risk of $\triangle \mathrm{TSS}>5$ exceeded $40 \%$. As joint damage is cumulative, we also assessed lower $\triangle \mathrm{TSS}$ thresholds. Risk of $\triangle \mathrm{TSS}>4$ was slightly greater than for $\Delta \mathrm{TSS}>5$, and the risk of $\Delta \mathrm{TSS}$ $>3$ or $>2$ exceeded $50 \%$ for the highest MBDA scores (Fig. 4b).

\section{Discussion}

This study has validated the adjusted MBDA score as a prognostic test for radiographic progression in RA using two cohorts that have not been evaluated previously with the adjusted MBDA score. These cohorts were then combined with two others for the largest analysis to date of the adjusted MBDA score as a prognostic test for radiographic progression. These cohorts came from two registries and two clinical trials and were diverse in their levels of clinical disease activity and treatments, which

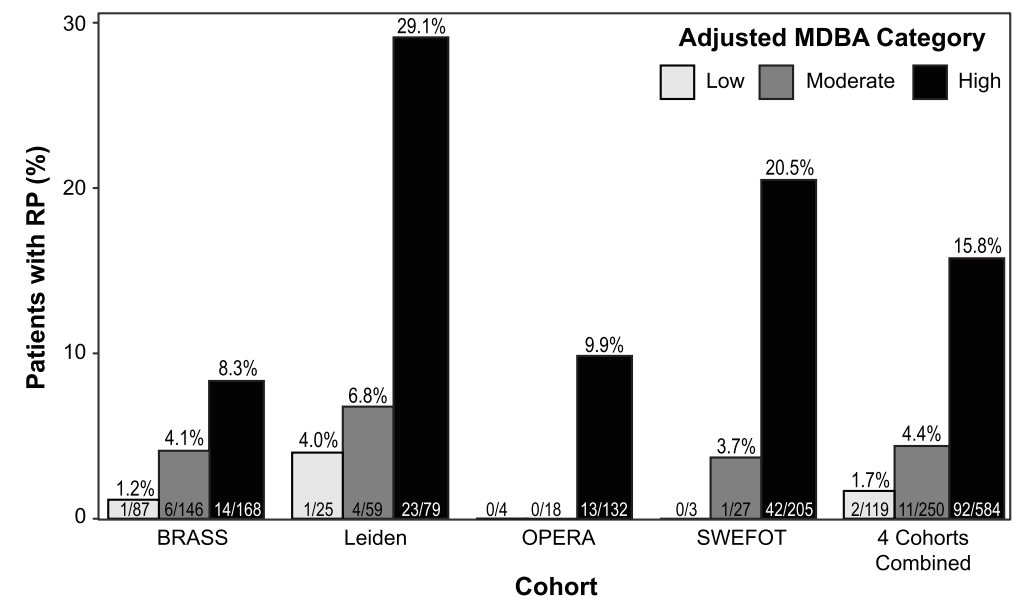

Fig. 2 Percentages of patients with radiographic progression (RP) by category of adjusted MBDA score. Results are shown for the Leiden, OPERA, SWEFOT, and BRASS cohorts individually and for the 4 cohorts combined. Radiographic progression was defined as change in van der Heijdemodified Sharp score (ATSS) > 5 units per year. Overall, 105 of 953 (11\%) patients progressed. MBDA multi-biomarker disease activity 


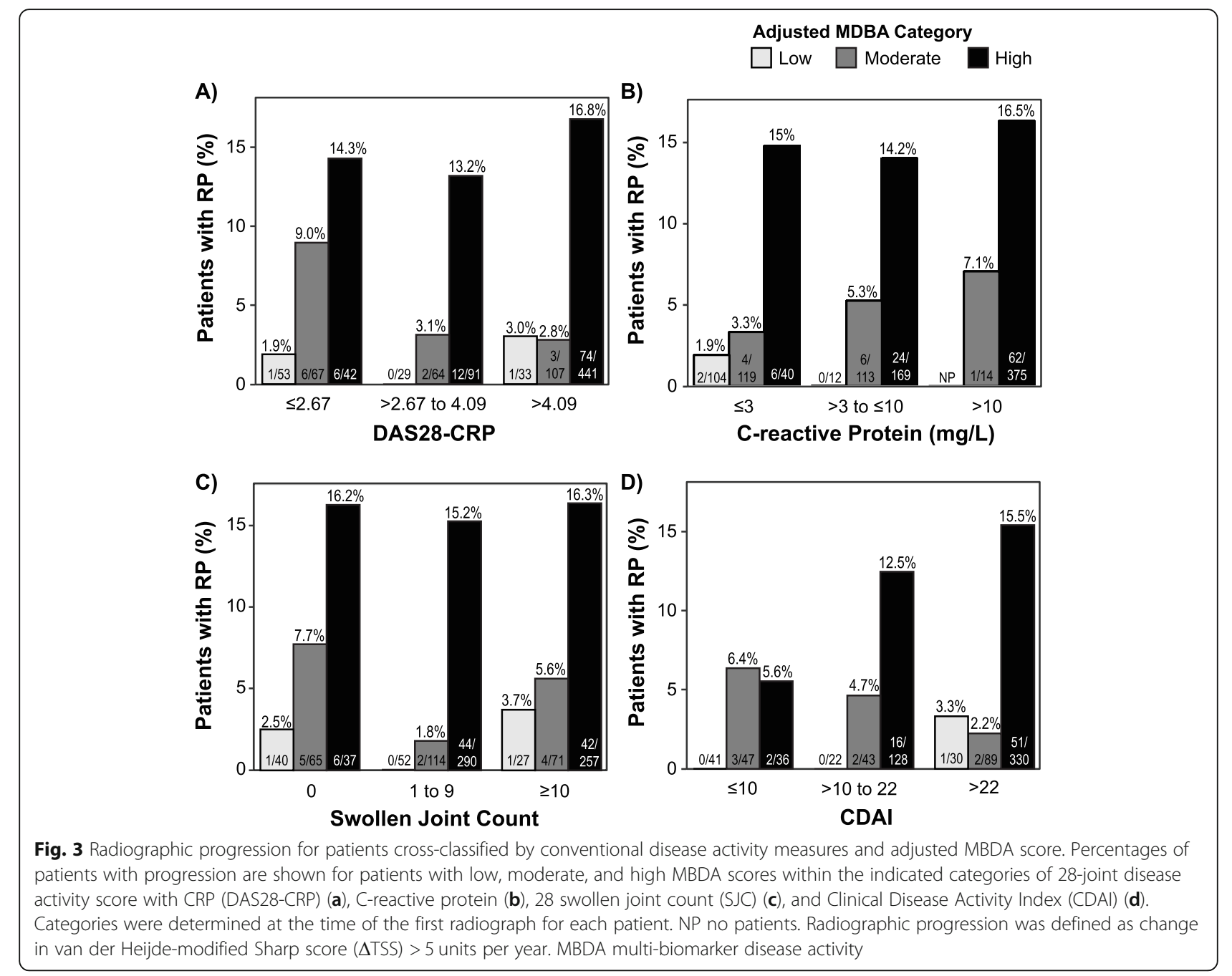

A)

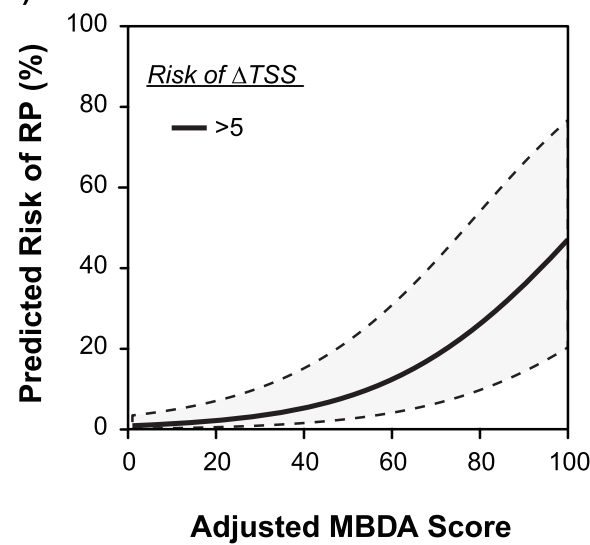

B)

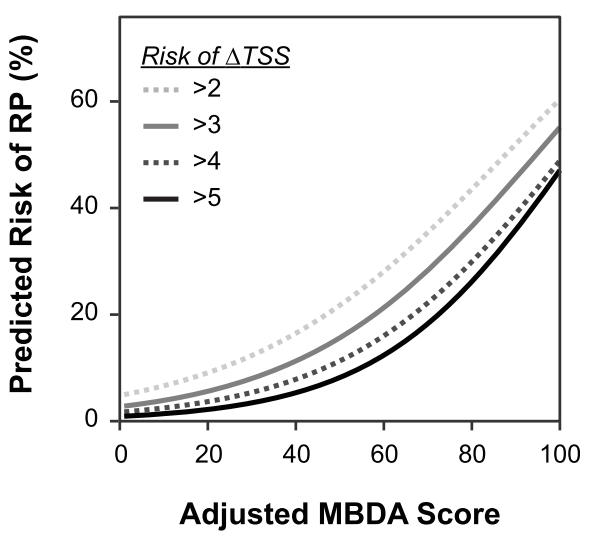

Fig. 4 Curves for predicting risk of radiographic prediction as a function of the adjusted MBDA score as a continuous variable. Progression was defined as change $(\Delta)$ in van der Heijde-modified Sharp score (TSS) $>5$ units per year and shown with $95 \%$ confidence interval (a), and as $\Delta$ TSS $>2,3$, 4 , and 5 units per year $(\mathbf{b})$. Curves were determined by logistic regression in the combined cohort $(N=953)$. MBDA multi-biomarker disease activity 
included non-biologic and biologic DMARDs (Table 1). The study produced, for the first time, a risk curve showing the probability of rapid radiographic progression $(\triangle \mathrm{TSS}>5)$ across the spectrum of adjusted MBDA scores. A low-adjusted MBDA score $(<30)$ was associated with very low risk for radiographic progression, with risk increasing continuously as the MBDA score increased and exceeding $40 \%$ for patients with the highest MBDA scores.

The adjusted MBDA score was shown to be a more statistically significant predictor of radiographic progression than serologic status, baseline TSS and all of the RA disease activity measures we assessed. While statistically significant univariable differences do not themselves establish clinical meaning, neither DAS28-CRP, CRP, SJC, nor CDAI added significant prognostic ability when directly compared to the adjusted MBDA score in bivariable analyses, whereas the adjusted MBDA score added significant information in each comparison. This finding has two important implications. First, by demonstrating that the adjusted MBDA score may be used independently of clinical assessment to stratify risk for joint damage progression in individual RA patients, it implies that when the adjusted MBDA score is low, the current RA therapy should be adequate for preventing joint damage, whereas, when it is high, a more effective treatment regimen may be needed to reduce the risk for progression.

The second implication of the association between the adjusted MBDA score and radiographic progression is that it supports the adjusted MBDA score as an accurate measure of the type of inflammatory disease activity that damages joints, even when it is discordant with clinical assessments. This property was established with bivariable analyses and was illustrated with cross-classification analyses for both types of discordance: (1) high-adjusted MBDA score with low clinical disease activity, as was observed in $25 \%$ of patients with low DAS28-CRP and might occur in patients who have subclinical synovitis or are difficult to examine due to obesity or osteoarthritis and (2) low-adjusted MBDA score with high clinical disease activity, as was observed in $6 \%$ of patients with high DAS28-CRP and might occur in patients with fibromyalgia or other forms of non-inflammatory pain (Fig. 3a). Similar results were obtained when the adjusted MBDA score was compared with SJC or CDAI (Fig. 3c, d).

Many studies have found CRP to be a predictor of risk for radiographic progression [40], but its clinical utility is limited because it is often not elevated in patients with clinically active RA [20]. We found that the adjusted MBDA score was a stronger predictor of radiographic progression than CRP and that, in bivariable analyses, CRP added no independent information to the MBDA score. The adjusted MBDA score predicted risk for radiographic progression not only when CRP was low (< $3.0 \mathrm{mg} / \mathrm{L})$, but also when it was intermediate $(3-10 \mathrm{mg} /$ L) or higher $(>10 \mathrm{mg} / \mathrm{L}$ ) (Fig. 3b). This finding is novel and suggests that the superiority of the adjusted MBDA score over CRP as a prognostic for radiographic progression derives not only from its ability of to identify patients with inflammation in the lowest CRP group [34], but also to identify patients who, despite having an elevated CRP, have low risk for radiographic progression.

The CRP results suggest that the MBDA score detected patient heterogeneity that was independent of CRP, which may reflect the role of the 11 non-CRP biomarkers in the MBDA score algorithm [6, 22]. A prior analysis of the SWEFOT study showed that patients with baseline CRP $\leq 10 \mathrm{mg} / \mathrm{L}$ and a high baseline MBDA score $(>44)$ had comparable clinical and radiographic outcomes to those with CRP $>10 \mathrm{mg} / \mathrm{L}$ at baseline [41] The authors concluded that recruitment for trials requiring an elevated CRP may be enhanced by an enrollment criterion of CRP $>10 \mathrm{mg} / \mathrm{L}$ and/or MBDA score $>44$. The present findings provide additional evidence that the adjusted MBDA score may be complementary to CRP as an enrollment criterion.

A strength of this study is the diversity of the large combined cohort. By including patients with early and established RA and patients treated with non-biologic and biologic DMARDs, the results may be broadly applicable. Access to patient-level data allowed us to calculate adjusted MBDA scores for all four cohorts. The AMPLE study was not included here because patientlevel data were not available [42, 43]. However, a reanalysis of published data from AMPLE showed that the associations between the original MBDA score and radiographic progression in the abatacept and adalimumab arms were similar to associations observed in other studies and to what was seen here with the adjusted MBDA score [30, 44].

The purpose of the present study was to validate the MBDA score as a predictor of risk for radiographic progression and compare it with other measures. The ultimate goal of preventing radiographic progression is to maintain physical function, which was not evaluated in this study. The relationship between radiographic progression and physical function, which can be assessed with the Health Assessment Questionnaire (HAQ), typically requires observation over many years to become apparent. In 1-year observations, as analyzed here, HAQ score is more likely to be affected by changes in disease activity than by new joint damage, especially in studies of active, recent-onset RA, like OPERA and SWEFOT. Disease activity contributes to functional decline via a direct effect, from signs and symptoms, and an indirect effect, which is only partly mediated by radiographic joint damage [45]. The MBDA score has been shown to 
correlate with the HAQ score [23]. The relationship between MBDA score and subsequent change in HAQ score is potentially complex and could be an interesting subject for future investigation.

Other limitations of the present study are that radiographs were assessed by different readers in each cohort, patient global assessments were unavailable for the Leiden cohort, and, except for one patient, TNF inhibitors were the only biologic drugs included in the four cohorts. Data on smoking were not evaluated here [46], but a prior analysis of the SWEFOT cohort found that the original MBDA score was a strong independent predictor of progression $(\Delta \mathrm{TSS}>5)$ after adjusting for current smoking status [28].

\section{Conclusion}

In conclusion, we have validated the adjusted MBDA score and performed the largest combined analysis to date of it as a prognostic test for radiographic progression in RA. The adjusted MBDA score was a stronger predictor of radiographic progression than DAS28-CRP, CRP, SJC, and CDAI, and its prognostic ability was not improved by any of these other measures, including when it was discordant with them. A risk curve was generated to show that the risk of rapid radiographic progression approached zero when the adjusted MBDA score was low, and it increased continuously with the adjusted MBDA score, such that risk exceeded 40\% and included the most severe cases of progression when the adjusted MBDA score was very high. The results of this study validate the adjusted MBDA score as an objective, independent measure of disease activity that, without requiring information from clinical assessment, can stratify RA patients according to their risk for developing new joint damage.

\section{Supplementary Information}

The online version contains supplementary material available at https://doi. org/10.1186/s13075-020-02389-4

Additional file 1 : Supplemental Table 1. Bivariable logistic regression predicting radiographic progression $(\triangle \mathrm{TSS}>5$ ) using adjusted MBDA score with other predictors (DAS28-CRP, CDAI, SJC, seropositivity, baseline van der Heijde modified Sharp score [TSS], log[CRP+1]). Odds ratio (OR) was calculated with 95\% confidence interval (Cl) and p-value from likelihood ratio test. All models include a random effect on cohort. Supplemental Figure 1. Radiographic progression for patients in individual cohorts cross-classified by DAS28-CRP and adjusted MBDA score.

\section{Abbreviations}

MBDA: Multi-biomarker disease activity; RA: Rheumatoid arthritis; TSS: Total Sharp score; DAS28-CRP: Disease Activity Score with 28 joints using CRP; CRP: C-reactive protein; SJC: Swollen joint count; CDAl: Clinical Disease Activity Index; DMARD: Disease-modifying anti-rheumatic drug; ESR: Erythrocyte sedimentation rate; RF: Rheumatoid factor; CCP: Cyclic citrullinated peptide; MMP: Matrix metalloproteinase; Cl: Confidence interval; EAC: Early Arthritis Clinic; ABA: Abatacept; ADA: Adalimumab;

CS: Corticosteroids; HCQ: Hydroxychloroquine; IA: Intra-articular;
IR: Inadequate responder; MTX: Methotrexate; RT: Randomized trial; SSZ: Sulfasalazine

\section{Acknowledgements}

The authors thank Brooke Hullinger, JD, for her assistance preparing figures and tables and editing the manuscript.

\section{Authors' contributions}

$J R C, M E W, D D F, E H S$, and TWH contributed to the conception and design of the study. MEW, NAS, CHB, MO, MLH, SS, and TWH designed and/or conducted the clinical trials included in analyses, from which they contributed primary data. Design and performance of analyses were by $\mathrm{MH}$, $B M, D D F$, and EHS. JRC and EHS drafted the paper, with revisions for important intellectual content made by all authors. The authors gave the final approval of the submitted manuscript.

\section{Authors' information}

Eric Sasso, M.D., is an Affiliate Professor of Medicine (Rheumatology) at the University of Washington, Seattle, WA, USA.

\section{Funding}

This work was supported by Myriad Genetics, Inc.

\section{Availability of data and materials}

The datasets used and/or analyzed during the current study are available from the corresponding author on reasonable request.

\section{Ethics approval and consent to participate}

This work combined data from four parent studies or registries that had each previously obtained informed consent from participants and IRB approval. Data from each of these cohorts have been published previously.

\section{Consent for publication}

Not applicable

\section{Competing interests}

J Curtis received grants and personal fees from Abbvie, Amgen, BMS, Corrona, Eli Lilly, Jannsen, Myriad, Pfizer, Regeneron, Roche, and UCB during the conduct of the study. M Weinblatt received grants from Crescendo Bioscience during the conduct of the study and receives grants from Crescendo Bioscience, Bristol Myers Squibb, Sanofi, Lilly, Amgen; personal fees from Abbvie, Arena, Canfite, Corrona, GSK, Gilead, Horizon, Johnson and Johnson, Pfizer, Roche, Samsung, Bristol Myers Squibb, Sanofi/Regeneron, Lilly, Amgen; and other disclosures from Scipher, Set Point, Canfite Inmedix, and Lycera Vorso outside the submitted work. N Shadick received grants from Crescendo Bioscience during the conduct of the study and receives grants from Crescendo Bioscience, BMS, Amgen, Lilly, Sanofi, and Mallinckrodt and personal fees from BMS outside the submitted work. C Brahe has nothing to disclose. M Østergaard receives grants, personal fees, and non-financial support from AbbVie, grants, personal fees and nonfinancial support from BMS, personal fees from Boehringer-Ingelheim, personal fees from Eli Lilly, personal fees and non-financial support from Janssen, grants, personal fees, and non-financial support from Merck, personal fees and non-financial support from Pfizer, personal fees and non-financial support from Roche, grants, personal fees, and non-financial support from UCB, grants and personal fees from Celgene, personal fees from Sanofi, personal fees from Regeneron, grants, personal fees, and non-financial support from Novartis, personal fees from Orion, and personal fees from Hospira outside the submitted work. M Hetland received grants from Crescendo Bioscience during the conduct of the study and receives grants from BristolMyers Squibb, AbbVie, Roche, Novartis, Merck, Biogen, and Pfizer and personal fees from Merck, Biogen, Pfizer, Eli Lilly, Orion Pharma, CellTrion, and Samsung Bioepis outside the submitted work. S Saevarsdottir is a part-time employee of deCODE Genetics Inc., unrelated to this work. M Horton, B Mabey, D Flake, R Ben-Shachar, and E Sasso received personal fees and stock options from Myriad Genetics, Inc., during the conduct of the study and outside the submitted work. T. W. Huizinga receives grants and personal fees from Myriad Autoimmune. 


\section{Author details}

${ }^{1}$ University of Alabama at Birmingham, 510 20th Street S, Birmingham, AL, USA. ${ }^{2}$ Divison of Rheumatology, Inflammation and Immunity, Brigham and Women's Hospital, Boston, MA, USA. ${ }^{3}$ Copenhagen Center for Arthritis Research and DANBIO, Center for Rheumatology and Spine Diseases, Rigshospitalet, Valdemar Hansens vej 17, Glostrup, Denmark. ${ }^{4}$ Department of Clinical Medicine, University of Copenhagen, Blegdamsvej 3B, Copenhagen, Denmark. ${ }^{5}$ Division of Rheumatology and Clinical Epidemiology, Department of Medicine, Solna, Karolinska Institutet, SE-171 77 Stockholm, Sweden. ${ }^{6}$ Faculty of Medicine, School of Health Sciences, University of Iceland, Reykjavik, Iceland. ${ }^{7}$ Myriad Genetics, Inc., 320 Wakara Way, Salt Lake City, UT, USA. ${ }^{8}$ Crescendo Bioscience, Inc., 180 Kimball Way, South San Francisco, CA, USA. 'eiden University Medical Center, Albinusdreef 2, 2333 ZA Leiden, Netherlands.

Received: 3 September 2020 Accepted: 9 December 2020 Published online: 04 January 2021

\section{References}

1. Singh JA, Furst DE, Bharat A, Curtis JR, Kavanaugh AF, Kremer JM, et al. 2012 Update of the 2008 American College of Rheumatology recommendations for the use of disease-modifying antirheumatic drugs and biologic agents in the treatment of rheumatoid arthritis. Arthritis Care Res. 2012;64(5):625-39.

2. Smolen JS, Landewe R, Breedveld FC, Buch M, Burmester G, Dougados M, et al. EULAR recommendations for the management of rheumatoid arthritis with synthetic and biological disease-modifying antirheumatic drugs: 2013 update. Ann Rheum Dis. 2014;73(3):492-509.

3. Emery P, Genovese MC, van Vollenhoven R, Sharp JT, Patra K, Sasso EH. Less radiographic progression with adalimumab plus methotrexate versus methotrexate monotherapy across the spectrum of clinical response in early rheumatoid arthritis. J Rheumatol. 2009;36(7):1429.

4. Emery P, Fleischmann R, van der Heijde D, Keystone EC, Genovese MC, Conaghan PG, et al. The effects of golimumab on radiographic progression in rheumatoid arthritis: results of randomized controlled studies of golimumab before methotrexate therapy and golimumab after methotrexate therapy. Arthritis Rheum. 2011;63(5):1200-10.

5. Weinblatt ME, Schiff M, Valente R, van der Heijde D, Citera G, Zhao C, et al, Head-to-head comparison of subcutaneous abatacept versus adalimumab for rheumatoid arthritis: findings of a phase IIIb, multinational, prospective, randomized study. Arthritis Rheum. 2013;65(1):28-38.

6. Brahe $\mathrm{CH}, \varnothing$ stergaard $M$, Johansen JS, Defranoux N, Wang X, Bolce R, et al. Predictive value of a multi-biomarker disease activity score for clinical remission and radiographic progression in patients with early rheumatoid arthritis: a post-hoc study of the OPERA trial. Scand J Rheumatol. 2019;48(1): 9-16.

7. Genovese MC, van Adelsberg J, Fan C, Graham NMH, van Hoogstraten H, Parrino J, et al. Two years of sarilumab in patients with rheumatoid arthritis and an inadequate response to MTX: safety, efficacy and radiographic outcomes. Rheumatology. 2018;57(8):1423-31.

8. Smolen JS, Choe JY, Weinblatt ME, Emery P, Keystone E, Genovese MC, et al. Pooled analysis of TNF inhibitor biosimilar studies comparing radiographic progression by disease activity states in rheumatoid arthritis. RMD Open. 2020;6(1):e001096.

9. Harrold LR, Harrington JT, Curtis JR, Furst DE, Bentley MJ, Shan Y, et al. Prescribing practices in a US cohort of rheumatoid arthritis patients before and after publication of the American College of Rheumatology treatment recommendations. Arthritis Rheum. 2012;64(3):630-8.

10. Ramiro S, Landewé RBM, van der Heijde D, Sepriano A, FitzGerald O, Ostergaard M, Homik J, Elkayam O, Thorne JC, Larche M, Ferraciolli G, Backhaus M, Boire G, Combe B, Schaeverbeke T, Saraux A, Dougados M, Rossini M, Govoni M, Sinigaglia L, Cantagrel AG, Allaart CF, Barnabe C, Bingham CO, Tak PP, van Schaardenburg D, Hammer HB, Dadashova R, Hutchings E, Paschke J, Maksymowych WP. Is treat-to-target really working in rheumatoid arthritis? A longitudinal analysis of a cohort of patients treated in daily practice (RA BIODAM). Ann Rheum Dis. 2020;79(4):453-59.

11. de Hair MJH, Jacobs JWG, Schoneveld JLM, van Laar JM. Difficult-to-treat rheumatoid arthritis: an area of unmet clinical need. Rheumatology. 2017; 57(7):1135-44.

12. Brown AK, Conaghan PG, Karim Z, Quinn MA, Ikeda K, Peterfy CG, et al. An explanation for the apparent dissociation between clinical remission and continued structural deterioration in rheumatoid arthritis. Arthritis Rheum. 2008;58(10):2958-67.

13. van der Heijde DM. How to read radiographs according to the Sharp/van der Heijde method. J Rheumatol. 2000;27:261-3.

14. Bruynesteyn K, van der Heijde D, Boers M, Saudan A, Peloso P, Paulus H, et al. Determination of the minimal clinically important difference in rheumatoid arthritis joint damage of the Sharp/van der Heijde and Larsen/ Scott scoring methods by clinical experts and comparison with the smallest detectable difference. Arthritis Rheum. 2002;46(4):913-20.

15. Vastesaeger N, Xu S, Aletaha D, St Clair EW, Smolen JS. A pilot risk model for the prediction of rapid radiographic progression in rheumatoid arthritis. Rheumatology (Oxford). 2009;48(9):1114-21.

16. Curtis JR, Chen L, Danila MI, Saag KG, Parham KL, Cush JJ. Routine use of quantitative disease activity measurements among US rheumatologists: implications for treat-to-target management strategies in rheumatoid arthritis. J Rheumatol. 2018;45(1):40.

17. Pincus T. Advantages and limitations of quantitative measures to assess rheumatoid arthritis: joint counts, radiographs, laboratory tests, and patient questionnaires. Bull NYU Hosp Jt Dis. 2006;64(1 \& 2):32-9.

18. Sokka T, Pincus T. Erythrocyte sedimentation rate, C-reactive protein, or rheumatoid factor are normal at presentation in 35\%-45\% of patients with rheumatoid arthritis seen between 1980 and 2004: analyses from Finland and the United States. J Rheumatol. 2009;36(7):1387.

19. Wolfe F. The many myths of erythrocyte sedimentation rate and C-reactive protein. J Rheumatol. 2009;36(8):1568.

20. Kay J, Morgacheva O, Messing SP, Kremer JM, Greenberg JD, Reed GW, et al. Clinical disease activity and acute phase reactant levels are discordant among patients with active rheumatoid arthritis: acute phase reactant levels contribute separately to predicting outcome at one year. Arthritis Res Ther. 2014;16(1):R40.

21. Centola M, Cavet G, Shen Y, Ramanujan S, Knowlton N, Swan KA, et al. Development of a multi-biomarker disease activity test for rheumatoid arthritis. PLoS One. 2013;8(4):e60635.

22. Curtis JR, van der Helm-van Mil AH, Knevel R, Huizinga TW, Haney DJ, Shen $Y$, et al. Validation of a novel multibiomarker test to assess rheumatoid arthritis disease activity. Arthritis Care Res. 2012;64(12):1794-803.

23. Hirata S, Dirven L, Shen Y, Centola M, Cavet G, Lems WF, et al. A multibiomarker score measures rheumatoid arthritis disease activity in the BeSt study. Rheumatology. 2013;52(7):1202-7.

24. Chernoff D, Scott Eastman P, Hwang CC, Flake DD, Wang X, Kivitz A, et al. Determination of the minimally important difference (MID) in multi-biomarker disease activity (MBDA) test scores: impact of diurnal and daily biomarker variation patterns on MBDA scores. Clin Rheumatol. 2019;38(2):437-45.

25. Johnson TM, Register KA, Schmidt CM, O'Dell JR, Mikuls TR, Michaud K, et al. Correlation of the multi-biomarker disease activity score with rheumatoid arthritis disease activity measures: a systematic review and meta-analysis. Arthritis Care Res. 2019;71(11):1459-72.

26. England BR, Tiong BK, Bergman MJ, Curtis JR, Kazi S, Mikuls TR, et al. 2019 Update of the American College of Rheumatology recommended rheumatoid arthritis disease activity measures. Arthritis Care Res. 2019;71(12):1540-55.

27. Markusse IM, Dirven L, van den Broek M, Bijkerk C, Han KH, Ronday HK, et al. A multibiomarker disease activity score for rheumatoid arthritis predicts radiographic joint damage in the BeSt study. J Rheumatol. 2014;41(11): 2114-9.

28. Hambardzumyan K, Bolce R, Saevarsdottir S, Cruickshank SE, Sasso EH, Chernoff D, et al. Pretreatment multi-biomarker disease activity score and radiographic progression in early RA: results from the SWEFOT trial. Ann Rheum Dis. 2015;74(6):1102-9.

29. Li W, Sasso EH, van der Helm-van Mil AH, Huizinga TW. Relationship of multi-biomarker disease activity score and other risk factors with radiographic progression in an observational study of patients with rheumatoid arthritis. Rheumatology. 2016;55(2):357-66.

30. Curtis JR, Brahe $\mathrm{CH}$, Østergaard M, Lund Hetland M, Hambardzumyan K, Saevarsdottir $S$, et al. Predicting risk for radiographic damage in rheumatoid arthritis: comparative analysis of the multi-biomarker disease activity score and conventional measures of disease activity in multiple studies. Curr Med Res Opin. 2019;35(9):1483-93.

31. Curtis JR, Flake DD, Weinblatt ME, Shadick NA, Ostergaard M, Hetland ML, et al. Adjustment of the multi-biomarker disease activity score to account for age, sex and adiposity in patients with rheumatoid arthritis. Rheumatology. 2019;58(5):874-83. 
32. Inoue E, Yamanaka H, Hara M, Tomatsu T, Kamatani N. Comparison of Disease Activity Score (DAS)28 - erythrocyte sedimentation rate and DAS28C-reactive protein threshold values. Ann Rheum Dis. 2007;66(3):407-9.

33. Felson DT, Smolen JS, Wells G, Zhang B, van Tuyl LHD, Funovits J, et al. American College of Rheumatology/European League Against Rheumatism provisional definition of remission in rheumatoid arthritis for clinical trials. Arthritis Rheum. 2011;63(3):573-86.

34. Lee YC, Hackett J, Frits M, lannaccone CK, Shadick NA, Weinblatt ME, et al. Multibiomarker disease activity score and C-reactive protein in a crosssectional observational study of patients with rheumatoid arthritis with and without concomitant fibromyalgia. Rheumatology. 2016;55(4):640-8.

35. Lillegraven S, Paynter N, Prince FHM, Shadick NA, Haavardsholm EA, Frits $\mathrm{ML}$, et al. Performance of matrix-based risk models for rapid radiographic progression in a cohort of patients with established rheumatoid arthritis. Arthritis Care Res. 2013:65(4):526-33.

36. Visser K, Goekoop-Ruiterman YPM, de Vries-Bouwstra JK, Ronday HK, Seys PEH, Kerstens PJSM, et al. A matrix risk model for the prediction of rapid radiographic progression in patients with rheumatoid arthritis receiving different dynamic treatment strategies: post hoc analyses from the BeSt study. Ann Rheum Dis. 2010;69(7):1333.

37. Saevarsdottir S, Rezaei H, Geborek P, Petersson I, Ernestam S, Albertsson K, et al. Current smoking status is a strong predictor of radiographic progression in early rheumatoid arthritis: results from the SWEFOT trial. Ann Rheum Dis. 2015;74(8):1509-14.

38. Cook NR. Use and misuse of the receiver operating characteristic curve in risk prediction. Circulation. 2007;115(7):928-35.

39. Pepe MS, Kerr KF, Longton G, Wang Z. Testing for improvement in prediction model performance. Stat Med. 2013;32(9):1467-82.

40. Navarro-Compán V, Gherghe AM, Smolen JS, Aletaha D, Landewé R, van der Heijde D. Relationship between disease activity indices and their individual components and radiographic progression in RA: a systematic literature review. Rheumatology. 2014;54(6):994-1007.

41. van Vollenhoven RF, Bolce R, Hambardzumyan K, Saevarsdottir S, Forslind K, Petersson IF, et al. Brief report: enhancement of patient recruitment in rheumatoid arthritis clinical trials using a multi-biomarker disease activity score as an inclusion criterion. Arthritis Rheumatol. 2015;67(11):2855-60.

42. Fleischmann R, Connolly SE, Maldonado MA, Schiff M. Brief report: estimating disease activity using multi-biomarker disease activity scores in rheumatoid arthritis patients treated with abatacept or adalimumab. Arthritis Rheum. 2016;68(9):2083-9.

43. Fleischmann R, Connolly SE, Maldonado MA, Schiff M. Reply. Arthritis Rheumatol. 2017:69(4):867-868.

44. Curtis JR, Wright GC, Strand V, Davis CS, Hitraya E, Sasso EH. Reanalysis of the multi-biomarker disease activity score for assessing disease activity in the abatacept versus adalimumab comparison in biologic-naive rheumatoid arthritis subjects with background methotrexate study: comment on the article by Fleischmann et al. Arthritis Rheum. 2017. https://doi.org/10.1002/ art.39981.

45. Nair SC, Bijlsma JWJ, van der Werf JH, van der Veen MJ, Linn-Rasker SP, Vreugdenhil $S$, et al. Do radiographic joint damage and disease activity influence functional disability through different mechanisms? Direct and indirect effects of disease activity in established rheumatoid arthritis. J Rheumatol. 2013;40(9):1505-12

46. Finckh A, Dehler S, Costenbader KH, Gabay C. Swiss Clinical Quality Management project for RA. Cigarette smoking and radiographic progression in rheumatoid arthritis. Ann Rheum Dis. 2007;66(8):1066-71.

\section{Publisher's Note}

Springer Nature remains neutral with regard to jurisdictional claims in published maps and institutional affiliations.

\section{Ready to submit your research? Choose BMC and benefit from}

- fast, convenient online submission

- thorough peer review by experienced researchers in your field

- rapid publication on acceptance

- support for research data, including large and complex data types

- gold Open Access which fosters wider collaboration and increased citations

- maximum visibility for your research: over $100 \mathrm{M}$ website views per year

At BMC, research is always in progress.

Learn more biomedcentral.com/submissions 